\title{
Implantación de asignaturas de Building Information Modeling en las titulaciones de la ETSICCP
}

\begin{abstract}
Fco. Javier Camacho Torregrosa ${ }^{a}$, Víctor Martínez Ibáñez ${ }^{b}$ José Ángel Aranda Domingo $^{c}$ y Alberto Domingo Cabo ${ }^{d}$

${ }^{a}$ Departamento de Ingeniería e Infraestructura de los Transportes, Universitat Politècnica de València. Email: fracator@tra.upv.es; ${ }^{b}$ Departamento de Ingeniería del Terreno, Universitat Politècnica de

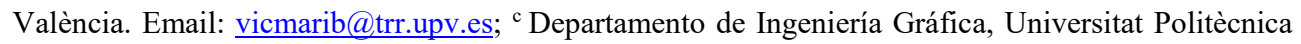
de València. Email: jaranda@dig.upv.es; ${ }^{d}$ Departamento de Ingeniería de la Construcción y de Proyectos de Ingeniería Civil, Universitat Politècnica de València. Email: adoming@,cst.upv.es.
\end{abstract}

\begin{abstract}
Building Information Modeling (BIM) establishes a dramatic improvement over construction projects, both for building and civil engineering. Its use is rapidly growing among professionals, especially for buildings.

To prepare students as best as possible for their further professional career, this methodology must be included in civil engineering studies (both bachelor and master). However, there are only a few of them already including this topic. Valencian Civil Engineering School (ETSICCP) decided to include an optional subject in four studies related to civil engineering (two bachelors, two masters), starting in the academic year 2018/19.

This paper develops the contents and teaching methodology for all four subjects, highlighting the opportunities and challenges, as well as the first results attained.
\end{abstract}

Keywords: Building Information Modeling, BIM, civil engineering, construction,

\footnotetext{
Resumen

La metodología Building Information Modeling (BIM) supone un importante avance cualitativo en el desarrollo de proyectos constructivos, tanto de edificación como de ingeniería civil. Esta metodología se está abriendo paso rápidamente en el ámbito profesional, especialmente en edificación.

Con el fin de preparar lo mejor posible al alumnado de cara a su desarrollo profesional, se hace indispensable incluir esta metodología dentro de los estudios relacionados con la ingeniería civil. Sin embargo, son muy pocos los grados o másteres habilitantes relacionados que cubran estas necesidades. La ETSICCP tomó la decisión de incorporar cuatro asignaturas plenamente enfocadas al trabajo en BIM en cuatro titulaciones diferentes (tres de ellas habilitantes), impartiéndose por primera vez en el curso 2018/19.
} 
En esta comunicación se presenta el desarrollo de contenidos y metodología docente para estas cuatro asignaturas, destacando las oportunidades y dificultades en su implantación, así como los primeros resultados obtenidos.

Palabras clave: Building Information Modeling, BIM, ingeniería civil, construcción

\section{Introducción}

\subsection{Building Information Modeling}

El Building Information Modeling (BIM) es una metodología de trabajo colaborativa para la gestión de proyectos de edificación u obra civil a través de un modelo digital. Este modelo conforma una gran base de datos que permite gestionar los elementos que forman parte de la infraestructura durante todo el ciclo de vida de la misma (es.BIM a).

Este modelo supone un repositorio único con información ordenada, coordinada, coherente y compartida, que se actualiza en tiempo real durante las fases de la obra: proyecto, construcción y explotación (Martínez-Ibáñez, 2017a). Esto implica un gran ahorro de tiempo (no es necesario convertir o interpretar desde varios tipos de archivo), así como menor número de errores (además de su identificación más sencilla, la menor necesidad de intercambios de información reduce la frecuencia de errores).

El modelo anteriormente descrito no debe entenderse únicamente como un modelo 3D. A cada elemento se le pueden asociar numerosos metadatos, así como relaciones con otros componentes del modelo. Algunos de los metadatos permiten, de hecho, el desarrollo de modelos 4D (implementando el tiempo y proceso constructivo), 5D (gestión de costes), e incluso 6D (sostenibilidad) y 7D (operatividad) (Martínez-Ibáñez, 2017b).

La Directiva 2014/24/UE sobre contratación pública establece la necesidad de emplear sistemas electrónicos en procesos de contratación de obras, servicios y suministros a partir de septiembre de 2018. En España, la comisión es.BIM fijó como objetivo exigir la metodología BIM como requisito para la contratación en licitaciones de edificación en diciembre de 2018, y en licitaciones de infraestructuras en julio de 2019 (es.BIM b). El requisito de BIM es, por tanto, ya necesario.

En el sector de la edificación, la tecnología BIM está fuertemente implantada (y creciendo), pero no es el caso para la ingeniería civil. Ello ha provocado, además, que en la actualidad se disponga de un formato de intercambio (Industry Foundation Classes, IFC), válido para el intercambio de modelos BIM de edificación, pero no todavía maduro para funcionar con modelos de ingeniería civil (COSTIN, 2018).

\subsection{Situación de los estudios de BIM en las Escuelas de Caminos en España}

Siendo tan relevante la integración del BIM en el sector de la construcción, esta situación choca con el grado de implantación que goza en la actualidad en los grados y másteres habilitantes en España. Se han analizado los planes de estudios de 16 grados de Ingeniería 
Civil o Ingeniería de Obras Públicas (o similares) y 14 Másteres Universitarios en Caminos, Canales y Puertos ofertados en las universidades españolas (excluyendo la UPV), encontrando únicamente una asignatura optativa específicamente orientada hacia el BIM, y seis asignaturas (cinco optativas y una obligatoria) que, dentro de su desarrollo docente, incorporan el BIM en al menos un tema o una práctica, si bien no siendo parte troncal de la asignatura.

Al margen de las titulaciones examinadas, sí son más numerosos los másteres no habilitantes que versan sobre la metodología BIM, si bien la práctica totalidad de su contenido se centra en el sector de la edificación.

\subsection{Titulaciones impartidas en la ETSICCP}

La Escuela Técnica Superior de Ingeniería de Caminos, Canales y Puertos de la Universitat Politècnica de València imparte en la actualidad las siguientes titulaciones (disponibles en la web de la ETSICCP):

- Grado en Ingeniería Civil (GIC). Grado de cuatro años, habilitante para ejercer la profesión de Ingeniero Técnico en Obras Públicas, en su especialidad de Construcciones Civiles. Su Plan de Estudios está diseñado para que los alumnos puedan tener una continuidad natural al MICCP.

- Grado en Ingeniería de Obras Públicas (GIOP). Grado de cuatro años, habilitante para ejercer la profesión de Ingeniero Técnico de Obras Públicas, en sus tres especialidades (Construcciones Civiles, Hidrología, y Transportes y Servicios Urbanos).

- Máster en Ingeniería de Caminos, Canales y Puertos (MICCP). Máster de dos años, habilitante para ejercer la profesión de Ingeniero de Caminos, Canales y Puertos. Está especialmente diseñado para ser cursado por alumnos provenientes de GIC, si bien también permite el acceso desde GIOP mediante complementos de formación.

- Máster en Planificación y Gestión en la Ingeniería Civil (MAPGIC). Máster no habilitante, impartido en dos cursos académicos. Este máster profundiza en el conocimiento de planificación y gestión de proyectos, obras, infraestructuras y empresas en el sector de la construcción.

- Máster en Transporte, Territorio y Urbanismo (MATTU). Máster no habilitante, impartido en dos cursos académicos. El objetivo del máster es profundizar en el conocimiento de las materias de urbanismo, territorio, y sistemas de transporte.

- Máster en Ingeniería Ambiental (MIA). Máster no habilitante de dos cursos académicos, impartido conjuntamente con la Universitat de València. En sus estudios, se persigue formar técnicos capaces de compatibilizar el desarrollo de la sociedad con una perspectiva sostenible y respetuosa con el medio ambiente.

\section{Objetivos}

En la Introducción se ha planteado la relevancia del BIM en el sector de la construcción, y su estado actual de implementación en los estudios conducentes a las habilitaciones 
profesionales en España relacionadas con la ingeniería civil. Vista la falta de asignaturas relacionadas y dada su importancia en el futuro profesional más inmediato dentro de la ingeniería civil, la Escuela Técnica Superior de Ingeniería de Caminos, Canales y Puertos apostó por la creación de cuatro asignaturas cuatrimestrales optativas relacionadas, situándolas en cuatro titulaciones de las que imparte.

Dado el carácter transversal de estas asignaturas, la Escuela encargó el diseño de las asignaturas a cuatro departamentos diferentes: Ingeniería de la Construcción y de Proyectos en Ingeniería Civil, Ingeniería Gráfica, Ingeniería del Terreno e Ingeniería e Infraestructura de los Transportes.

Todas estas asignaturas reciben el mismo nombre ("Building Information Modeling (BIM)"), impartiéndose en los últimos cursos de las titulaciones de GIC, GIOP, MICCP y MAPGIC. El curso 2018/19 ha sido el primero en el que se han implantado las mismas, por lo que los resultados alcanzados no gozan de fortaleza estadística, aunque sí son prometedores.

El objetivo de esta comunicación es detallar el contenido desarrollado en cada una de ellas, el perfil de alumnado y las habilidades que se persigue que consiga tras cursar cualquiera de estas. A este objetivo se le contraponen importantes limitaciones, como:

1. Herramientas de trabajo. Es necesario que las asignaturas tengan un enfoque fundamentalmente práctico, por lo que se hace necesario elegir adecuadamente las herramientas informáticas necesarias. Si bien existen diversas opciones, es también muy importante que el alumno diferencie entre BIM (una metodología) y la herramienta informática a utilizar.

2. El entorno BIM, especialmente en el sector de la ingeniería civil, es muy cambiante a día de hoy. Esta evolución constante no solo requiere que los profesores estén actualizándose más rápidamente de lo habitual, sino que es necesario hacer a los alumnos conocedores de ello.

3. En las actuales titulaciones (especialmente las de grado), los alumnos han visto poco diseño de detalle en las asignaturas (están más orientadas hacia el cálculo). Por ello, será necesario impartir algunos temas de este tipo antes de diseñar en BIM.

4. Estando los alumnos en el último año de la titulación, resulta altamente recomendable que los contenidos desarrollados sirvan directamente al alumno para su aplicación en sus Trabajos Fin de Grado (TFG) o Trabajo Fin de Máster (TFM).

\section{Desarrollo de la innovación}

\subsection{Contenido teórico}

Como se ha indicado, la asignatura se implementó como optativa en las cuatro titulaciones siguientes, con el siguiente número de créditos y cuatrimestre:

- Grado de Ingeniería Civil (GIC). Semestre A, 4.5 créditos.

- Grado en Ingeniería de Obras Públicas (GIOP). Semestre B, 6 créditos. 
- Máster Universitario en Ingeniería de Caminos, Canales y Puertos (MICCP). Semestre B, 4.5 créditos.

- Máster Universitario en Planificación y Gestión en la Ingeniería Civil (MAPGIC). Semestre B, 3 créditos.

La variación en el número de créditos y el semestre viene condicionada por los bloques de optatividad establecidos para cada una de las titulaciones. Existe cierta flexibilidad en el momento de la impartición (semestre A o B), si bien en todos los casos se desarrolla en el último curso de la titulación.

Se decidió que las cuatro asignaturas debían compartir un tronco común de conocimientos, perfilándose en función de la titulación asociada. A continuación figuran las particularidades más destacadas dentro de cada titulación.

\subsubsection{Grado en Ingeniería Civil}

Los alumnos cursando el Grado de Ingeniería Civil adquirirán competencias para ejercer la profesión de Ingeniero Técnico de Obras Públicas (si bien únicamente para la especialidad de Construcciones Civiles). No obstante, tal y como se indicó anteriormente, este grado tiene como objetivo preparar a los estudiantes para cursar en las mejores condiciones posibles el Máster de Ingeniería de Caminos, Canales y Puertos, donde está localizada otra de las asignaturas BIM.

Puesto que los alumnos no tienen casi experiencia previa en el diseño de infraestructuras (básicamente en su cálculo), se considera recomendable que la asignatura de BIM contenga una primera parte de diseño detallado (no necesariamente bajo entorno BIM). Posteriormente, se complementarán con las nociones de diseño en BIM, sin llegar al máximo nivel de complejidad (que se reserva para MICCP).

\subsubsection{Grado en Ingeniería de Obras Públicas}

Los alumnos cursando este grado adquirirán también competencias habilitantes para ejercer de Ingenieros Técnicos de Obras Públicas, en las tres especialidades. Sin embargo, la estructura de su Plan de Estudios no está concebida para tener continuidad en el Máster de Ingeniería de Caminos (si bien tampoco lo impide). Así pues, esta asignatura se plantea más finalista. De hecho, es la que cuenta con mayor número de créditos (6), facilitando este objetivo.

Al igual que en el caso del GIC, los alumnos tienen poca formación previa en diseño de detalle de infraestructuras, por lo que vuelve a ser necesario contar con una introducción similar. Sin embargo, se considera también necesario impartir una serie de conceptos teóricos de refuerzo de diseño de infraestructuras lineales, que sí había sido visto por los alumnos de GIC en otras asignaturas.

Los alumnos de GIOP deben ser capaces de generar modelos BIM sencillos, así como de explorar modelos generados por otras personas. Por ello, se prefiere abarcar más tipos de elementos de diseño (como señales verticales, barreras de seguridad, drenaje, etc.), en vez de dar un diseño de detalle (como por ejemplo diseñar intersecciones). 


\subsubsection{Máster en Ingeniería de Caminos, Canales y Puertos}

Los alumnos que cursen esta versión de la asignatura persiguen la adquisición de competencias para ejercer la profesión de Ingeniero de Caminos, Canales y Puertos. Por ello, es en esta asignatura cuando se desarrollan los contenidos con mayor profundidad.

A nivel de conocimientos previos, casi la totalidad de alumnos habrán cursado GIC, así como las asignaturas asociadas al propio máster. Con todo ello, se considera que a nivel técnico los alumnos están suficientemente formados.

Además, los alumnos ya han tenido la posibilidad de cursar una de las otras asignaturas BIM en las anteriores titulaciones (GIC o GIOP). Esto supone a su vez una ventaja y un inconveniente. Por una parte, el que muchos alumnos ya conozcan la metodología permite establecer un punto de partida para alcanzar una mayor profundidad en la asignatura. Por otra, diferencia las habilidades de partida de los alumnos.

\subsubsection{Máster Universitario en Planificación y Gestión en Ingeniería Civil}

Este máster no es habilitante, por lo que los objetivos finales de la asignatura deberán plantearse en función del resto de asignaturas y objetivos del Máster en cuestión. Asimismo, los conocimientos previos de los alumnos son mucho más diversos.

Con estos condicionantes, se eligió prescindir de un diseño detallado en BIM, centrándose (tal y como enuncia el título del máster) más en la planificación a nivel 4D y la gestión de costes 5D.

\subsubsection{Estructura de las asignaturas}

Tomando como base las restricciones anteriores, se diseñaron las asignaturas con una base teórica común y diez prácticas. La base teórica de BIM se ha planteado igual para todas las asignaturas, con los siguientes temas:

1. Introducción al BIM.

2. Del CAD al BIM.

3. La implantación del BIM en la construcción.

4. Trabajo colaborativo. Disciplinas integradas en un proyecto y herramientas de interoperabilidad.

5. BIM Execution Plan (BEP).

Los detalles de las sesiones prácticas pueden verse en la Tabla 1. Para cada práctica se detalla el número de horas y los contenidos desarrollados. Ello permite diferenciar entre las versiones de asignaturas para cada titulación. 
Tabla 1. Distribución de prácticas en las asignaturas, e indicadores de sus contenidos

\begin{tabular}{|c|c|c|c|c|}
\hline & GIC & GIOP & MICCP & MAPGIC \\
\hline P1. Manejo de cartografía & $5 \mathrm{~h}$ & $4 \mathrm{~h}$ & $3 \mathrm{~h}$ & $2 \mathrm{~h}$ \\
\hline \multicolumn{5}{|l|}{ Conocer las fuentes públicas de obtención de datos } \\
\hline \multicolumn{5}{|l|}{ Distinguir entre $M D T$ y $M D E$} \\
\hline \multicolumn{5}{|l|}{ Crear una superficie a partir de una nube de puntos } \\
\hline \multicolumn{5}{|l|}{ Modos de visualización de superficies } \\
\hline \multicolumn{5}{|l|}{ Análisis de superficies } \\
\hline \multicolumn{5}{|l|}{ Operaciones con superficies } \\
\hline P2. Generación de alineaciones en planta y alzado & $6 \mathrm{~h}$ & $8 \mathrm{~h}$ & $4 \mathrm{~h}$ & $4 \mathrm{~h}$ \\
\hline \multicolumn{5}{|l|}{ Refuerzo teórico de diseño de carreteras } \\
\hline \multicolumn{5}{|l|}{ Creación de ejes en planta y alzado } \\
\hline \multicolumn{5}{|l|}{ Definición de una sección transversal simple } \\
\hline \multicolumn{5}{|l|}{ Definición de una sección transversal compleja } \\
\hline \multicolumn{5}{|l|}{ Análisis de visibilidad y de movimientos de tierras } \\
\hline \multicolumn{5}{|l|}{ Disposición de elementos de drenaje } \\
\hline P3. Creación de intersecciones y enlaces & $4 \mathrm{~h}$ & & $4 \mathrm{~h}$ & \\
\hline \multicolumn{5}{|l|}{ Diseño por envolventes con Vehicle Tracking } \\
\hline \multicolumn{5}{|l|}{ Ajuste de una intersección en $T$} \\
\hline \multicolumn{5}{|l|}{ Ajuste de una glorieta } \\
\hline \multicolumn{5}{|l|}{ Diseño de enlaces } \\
\hline P3. Señalización y balizamiento. Marcas viales & & $7 \mathrm{~h}$ & & \\
\hline \multicolumn{5}{|l|}{ Refuerzo teórico } \\
\hline \multicolumn{5}{|l|}{ Disposición de barreras de seguridad } \\
\hline \multicolumn{5}{|l|}{ Disposición de señalización vertical y balizamiento } \\
\hline \multicolumn{5}{|l|}{ Disposición de señalización horizontal } \\
\hline P4. Modelado de un puente & $4 \mathrm{~h}$ & $3 \mathrm{~h}$ & $4 \mathrm{~h}$ & $2 \mathrm{~h}$ \\
\hline \multicolumn{5}{|l|}{ Introducción de tipologías de estructuras en puentes } \\
\hline \multicolumn{5}{|l|}{ Selección de la estructura adecuada } \\
\hline \multicolumn{5}{|l|}{ Diseño del puente: tablero, vigas, pilas y estribos } \\
\hline \multicolumn{5}{|l|}{ Visualización del modelo } \\
\hline \multicolumn{5}{|l|}{ Análisis del modelo generado respecto al terreno } \\
\hline Exportación de la estructura (IFC) & & & & \\
\hline P5. Exportación de formatos. Modelos federados & $2 \mathrm{~h}$ & $3 \mathrm{~h}$ & $2 \mathrm{~h}$ & $2 \mathrm{~h}$ \\
\hline Concepto de federación & & & & \\
\hline Exportación de Civil 3D / Istram a Navisworks & & & & \\
\hline Análisis de secciones & & & & \\
\hline Revisión del modelo & & & & \\
\hline Análisis de colisiones & & & & \\
\hline P6. Planificación 4D & $2 \mathrm{~h}$ & $3 \mathrm{~h}$ & $2 \mathrm{~h}$ & $2 \mathrm{~h}$ \\
\hline Organizar unidades de ejecución y estimar duraciones & & & & \\
\hline Preparar diagrama de Gantt. Introducción a MS Project & & & & \\
\hline Asignación de objetos del modelo al programa de trabajos & & & & \\
\hline Crear simulación del proceso constructivo. Exportar vídeo & & & & \\
\hline Análisis de los datos de programación asignados a objetos & & & & \\
\hline
\end{tabular}




\begin{tabular}{|l|l|l|l|l|}
\hline $\begin{array}{l}\text { P7. Programación 5D. Mediciones, presupuestos y } \\
\text { gestión de costes }\end{array}$ & $2 \mathrm{~h}$ & $3 \mathrm{~h}$ & $2 \mathrm{~h}$ & $3 \mathrm{~h}$ \\
\hline Identificar si los objetos contienen datos de medición & & & & \\
\hline Creación y configuración de un libro de cuantificación & & & & \\
\hline Extracción de datos de medición a un libro de cuantificación & & & & \\
\hline Exportación y revisión de las mediciones completas & & & & \\
\hline Importar libro de cuantificación a software de presupuestos & & & & \\
\hline P8. Realismo & $2 \mathrm{~h}$ & $3 \mathrm{~h}$ & $2 \mathrm{~h}$ & $2 \mathrm{~h}$ \\
\hline Creación de escenarios. & & & & \\
\hline Importación de modelos IFC, y de otros formatos nativos & & & & \\
\hline Análisis del terreno y de los objetos BIM & & & & \\
\hline Modelado de carreteras: planta, alzado y ensamblajes & & & & \\
\hline Creación de intersecciones & & & & \\
\hline Modelado de puentes y túneles & & & & \\
\hline Inserción de obras de drenaje y su comprobación hidráulica & & & & \\
\hline Revisión y publicación del modelo & & & & \\
\hline P9. Introducción a Revit & $2 \mathrm{~h}$ & $3 \mathrm{~h}$ & $2 \mathrm{~h}$ & $2 \mathrm{~h}$ \\
\hline Introducción a la interface de Revit & & & & \\
\hline Creación de una plantilla en Revit & & & & \\
\hline Importar ficheros dwg & & & & \\
\hline Introducción de niveles & & & & \\
\hline Creación del terreno & & & & \\
\hline Creación de una plataforma de construcción & & & \\
\hline P10. Generación de un modelo de arquitectura & & $3 \mathrm{~h}$ & $2 \mathrm{~h}$ & $2 \mathrm{~h}$ \\
\hline Masas conceptuales & & & & \\
\hline Envolvente de una vivienda & & & \\
\hline Dibujo de elementos constructivos & & & \\
\hline Creación de Suelos & & & \\
\hline Creación de Techos & & & \\
\hline
\end{tabular}

\subsection{Metodología docente}

Debido al fuerte carácter profesional de la asignatura, su ámbito práctico debe estar presente en gran medida desde el inicio. Es por ello que más del 50\% de todo su contenido se desarrolla mediante prácticas de informática, o combinando teoría y práctica de informática en la misma aula.

El objetivo de las asignaturas no es mostrar cómo se realiza cada paso con ciertas aplicaciones informáticas, sino mostrar qué es lo que se quiere hacer, y cómo se utiliza una determinada herramienta para ello. En resumen, separar el "qué" del "cómo". Esto, entendido adecuadamente por los alumnos, les permitirá utilizar otra herramienta para el mismo fin, en un futuro profesional próximo y altamente cambiante.

Para cada una de las prácticas de la asignatura, los alumnos deben realizar unos entregables, generalmente consistentes en el diseño de una parte de una infraestructura, o un modelo sencillo. El profesor muestra en clase los pasos a realizar (con otro ejemplo), y son los 
alumnos (individualmente, en parejas o en grupos, según la práctica) los que posteriormente desarrollan su caso particular. La entrega generalmente debe realizarse al finalizar la clase, o con un pequeño margen de tiempo de uno o dos días.

PoliformaT es la herramienta propia de la Universitat Politècnica de València para la compartición de contenidos entre profesores y alumnos. En ella, los profesores pueden difundir sus materiales docentes a los alumnos, y estos últimos pueden entregar las prácticas, hacer exámenes online, etc.

Las asignaturas tan prácticas pueden tener problemas importantes en el seguimiento por parte de los alumnos. Algunos factores influyentes son su diferente grado de relación con la tecnología o la rapidez de procesamiento de los ordenadores. Esto puede implicar un retraso en el desarrollo lectivo e incluso no llegar a cubrir los objetivos.

Con el fin de minimizar estos potenciales problemas, así como de facilitar el aprendizaje autónomo por parte de los alumnos, se decidió crear un gran número de recursos de aprendizaje multimedia para la asignatura (más de 50 vídeos). En su mayoría, estos recursos fueron vídeos didácticos screencast, de entre 5 y 15 minutos de duración, con los diferentes pasos que se enseñaron también en clase. Aunque formalmente la asignatura no ha sido planteada como en modalidad de docencia inversa, estos materiales sí permitirían el seguimiento de este modo. De hecho, los vídeos son materiales con muy buena recepción por parte de los alumnos (Segura et al., 2018). Estos permiten particularizar el ritmo de aprendizaje a las demandas de cada alumno, más allá de la pura memorización, y desarrolla otras habilidades como colaboración, comunicación y resolución de problemas (VasilevaStojanovska et al., 2015).

Para facilitar la organización de tal cantidad de recursos, se empleó la herramienta LESSONS, disponible dentro de PoliformaT. Esta herramienta permite configurar los contenidos de una asignatura como si de una web se tratase (con hipervínculos, contenido incrustado y scripts interactivos). Un ejemplo puede verse en la Figura 1. Además de los contenidos teóricos, cada uno de estos LESSONS enlazaba a los ejercicios de cada tema, manuales desarrollados por los profesores, pequeñas preguntas de autoevaluación, e incluso foros de discusión. 


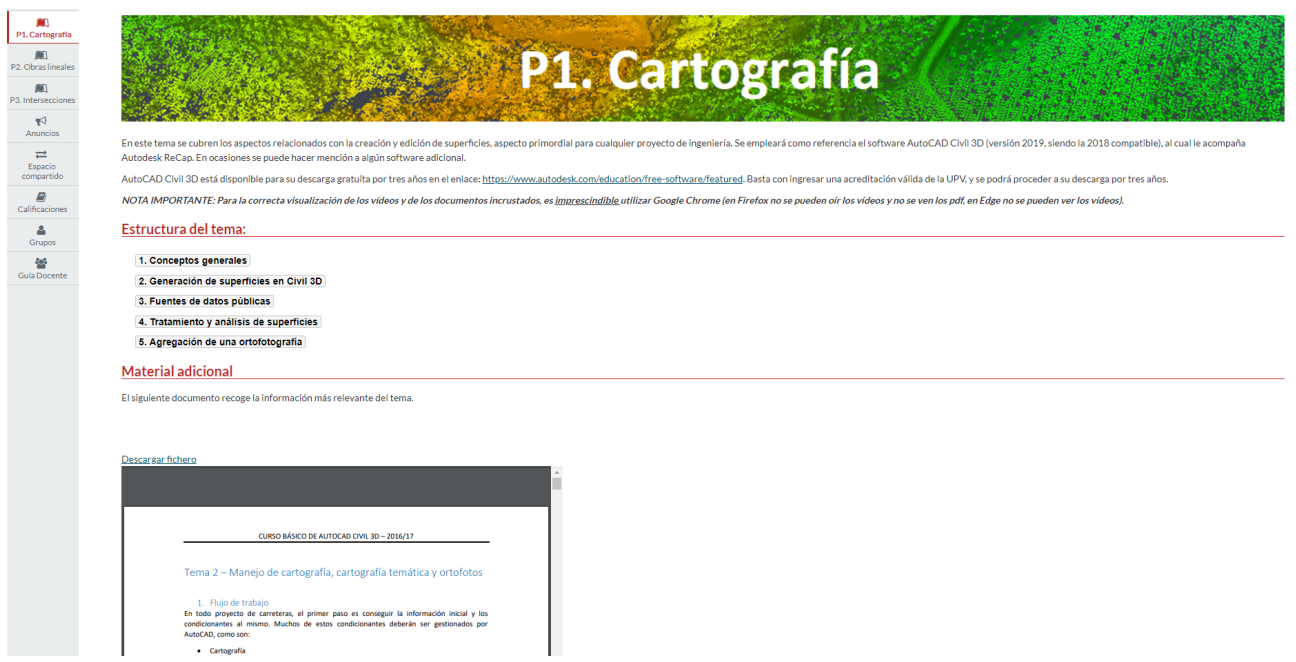

Fig. 1. Ejemplo de un tema desarrollado en LESSONS, con enlaces a otras páginas del mismo y un manual (descargable y visualizable en pantalla).

\subsection{Otros aspectos}

En este epígrafe se comentan otros aspectos de importancia en el planteamiento de las asignaturas.

\subsubsection{Evaluación}

La evaluación - con pequeñas variaciones en los pesos de cada parte - se basa en los siguientes tres ámbitos:

1. Examen ordinario, tipo test, sobre los aspectos teóricos vistos en clase.

2. Evaluación de los ejercicios que entregan al finalizar cada práctica.

3. Entrega de un vídeo, de cinco a diez minutos de duración y ejecutado en grupos de cuatro alumnos, en los que sintetizan lo que han aprendido en el curso. Este vídeo también permite la evaluación de la competencia transversal de comunicación oral.

\subsubsection{Aplicaciones utilizadas}

La elección del software fue un tema abordado con detalle en la preparación de la asignatura. Existen en el mercado diferentes posibilidades. Si bien cada software puede presentar pequeñas ventajas o inconvenientes, no existe un claro dominador en el mercado que haga inclinar la balanza. En la decisión se consideraron factores como la interacción con otro software existente, disponibilidad de licencias, expansión nacional e internacional y requisitos de hardware. Asimismo, se quería a toda costa evitar que los alumnos identificaran el BIM con un software en particular.

Finalmente, se tomó la decisión de implementar en mayor medida el software AutoCAD Civil 3D (de Autodesk, Inc.) para GIC y MICCP, e ISTRAM (de Buhodra Ingeniería S.A.) en GIOP y MAPGIC. Para federar modelos se empleó Navisworks, para su visualización en 
modelos colaborativos, Infraworks, y para el desarrollo de modelos de arquitectura, Revit, todos ellos de Autodesk Inc. Este software podrá variar en futuros cursos académicos, en función de como cambie su implantación en el mercado, o sus posibilidades técnicas.

\subsubsection{Integración con la práctica profesional}

Uno de los mayores atractivos de la asignatura consiste en su fuerte relación con el desarrollo profesional de los alumnos. Con el fin de integrarlo con la práctica profesional, dar continuidad y actualizar el contenido de la asignatura, se complementó con la impartición de charlas realizadas por profesionales especialistas en el ámbito.

\section{Resultados}

Siendo el 2018/19 el primer curso de impartición de las asignaturas, los resultados disponibles son bien limitados. La asignatura del GIC se desarrolló en el primer semestre, lo que permitió extraer ciertas conclusiones para su aplicación en las otras tres, del segundo semestre.

La acogida de la asignatura ha sido muy favorable, a tenor de los datos de matrícula y comparando con el número de egresados (último dato conocido, del curso 2017/18) (Tabla 2).

Tabla 2. Distribución de prácticas en las asignaturas, e indicadores de sus contenidos

\begin{tabular}{|l|c|c|}
\hline Titulación & Matrícula & Egresados curso 2017/18 \\
\hline Grado en Ingeniería Civil & 39 & 71 \\
\hline Grado en Ingeniería de Obras Públicas & 25 & 74 \\
\hline $\begin{array}{l}\text { Máster en Ingeniería de Caminos, Canales } \\
\text { y Puertos }\end{array}$ & 20 & 23 \\
\hline $\begin{array}{l}\text { Máster en Planificación y Gestión en la } \\
\text { Ingeniería Civil }\end{array}$ & 24 & \\
\hline
\end{tabular}

La asignatura tuvo asimismo una buena acogida para alumnos provenientes de otras universidades. De hecho, ocho de los alumnos en la asignatura de GIC eran extranjeros, cinco de los cuales además no hablaban español de forma nativa. Esto no fue una limitación para impartir las clases, más bien al contrario, viéndose como una oportunidad para ofertar la asignatura en lengua extranjera (inglés) en próximos cursos.

Asimismo, la disposición de un gran número de materiales multimedia en la web de la asignatura fue muy útil para los alumnos, pues pudieron consolidar los contenidos vistos en clase, e incluso ampliarlos. De hecho, algunos alumnos refirieron que estos materiales eran especialmente útiles para su visualización antes de la clase práctica (a modo de docencia inversa), lo cual también fue apreciado por los profesores al comparar su rendimiento en las 
prácticas. Con el objetivo de profundizar en mayor medida en este aspecto, se decidió impartir algunos de los temas de las otras tres asignaturas directamente en modalidad de docencia inversa.

Es asimismo reseñable que varios alumnos están desarrollando sus Trabajos Fin de Grado mediante las metodologías mostradas en la asignatura, especialmente en los diseños previos (mediante Infraworks) y la producción de detalle (Civil o Istram).

En futuros cursos académicos, es de esperar que gran parte de los alumnos que escojan la asignatura en los Másteres sea porque ya han cursado la optativa en el grado correspondiente. Sin embargo, esta posibilidad no podrá darse hasta el curso 2020/21. Hasta entonces, ninguno de los alumnos de máster tiene experiencia previa en BIM (impartida en titulaciones de la Escuela). Por ello, los profesores decidieron rebajar los objetivos de la asignatura del MICCP los cursos 2018/19 y 2019/20, haciéndolos más similares a la asignatura de GIC.

A partir del curso 2020/21, la asignatura de BIM del MICCP contará con toda la profundidad aquí expuesta, pudiendo ser un problema potencial para los alumnos que no cursasen anteriormente la optativa en GIC (o, en menor medida, GIOP). Para reducir este impacto, se prevé la creación de una lección de nivelación, también en la plataforma LESSONS, que pueda servir para homogeneizar de algún modo los contenidos más prácticos.

\section{Conclusiones}

En esta comunicación se han mostrado el desarrollo docente de cuatro asignaturas relacionadas con el Building Information Modeling (BIM), habilitadas por primera vez el curso 2018/19 en cuatro titulaciones de la ETSICCP.

Los contenidos teóricos de todas ellas se centran en un tronco común, con diferentes niveles de complejidad según la titulación, habilidades previas de los alumnos, y competencias que deben adquirir. Asimismo, se presentan las principales dificultades o limitaciones encontradas tras impartir una de estas asignaturas.

Estas asignaturas permitirán una mayor preparación del alumno con vistas a su futuro profesional. De hecho, futuras ediciones de la asignatura también variarán en función de la evolución del BIM en el sector de la ingeniería civil.

\section{Referencias}

COSTIN, A.; ADIBFAR, A.; HU, H. and CHEN, S.S. (2018). "Building Information Modeling (BIM) for transportation infrastructure - Literature review, applications, challenges, and recommendations". Automation in Construction, 94. pp. 257-281.

Diario Oficial de la Unión Europea. Directiva 2014/24/UE sobre contratación pública $<$ https://www.boe.es/doue/2014/094/L00065-00242.pdf> [Consulta 24 de marzo de 2019]

es.BIM a. Sobre BIM https://www.esbim.es [Consulta: 20 de marzo de 2019] 
es.BIM b. $4^{\text {a }}$ reunión de la Comisión BIM. <http://www.esbim.es/wpcontent/uploads/2016/08/esBIM-Cuarta-reunion.pdf> [Consulta: 23 de marzo de 2019]

ETSICCP. Titulaciones impartidas en la ETSICCP $<$

http:/www.cam.upv.es/web/expl.aspx?id=\$2\$Estudiantes $\$ 1 \$$ Titulaciones $>$ [Consulta: 24 de marzo de 2019]

MARTÍNEZ-IBÁÑEZ, V. Y PELLICER, E. (2017a). "Propuesta metodológica para la implementación de la información geotécnica en modelos BIM”. Congreso Internacional BIM. Valencia, mayo de 2017.

MARTÍNEZ-IBÁÑEZ, V.; PELLICER, E.; ALCOBENDAS, J. Y CASADO, S. (2017b). "Retos en la implantación de BIM en la ingeniería civil y propuestas para acelerar su aplicación”. Congreso Internacional BIM. Valencia, mayo de 2017.

SEGURA, M.; MAROTO, C.; GINESTAR, C: y NAVARRO, J.R. (2018). "Preferencias y rendimiento de los alumnos en el aprendizaje de Investigación Operativa con vídeos". IV Congreso Nacional de Innovación Educativa y Docencia en Red (INRED). Valencia, junio de 2018.

VASILEVA-STOJANOVSKA, T.; MALINOVSKI T.; DOBRIJOVEVSKI, M.V. y TRAJKOVIK, V. (2015) "Impact of satisfaction, personality and learning style on educational outcomes in a blended learning environment". Learning and Individual Differences 38, pp. 127-135. 